\title{
Rapid Eye Movement Sleep Behavior Disorder
}

\author{
Sooyeoun You, Soo Myeong Jeon, Yong Won Cho \\ Department of Neurology, Dongsan Medical Center, Keimyung University School of Medicine, Daegu, Korea
}

\section{렘수면 행동 장애}

유수연, 전수명, 조용원

계명대학교 의과대학 신경과학교실

Received January 10, 2018 Revised January 24, 2018

Accepted January 26, 2018

Address for correspondence Yong Won Cho, MD, PhD

Department of Neurology, Dongsan Medical Center,

Keimyung University

School of Medicine,

56 Dalseong-ro, Jung-gu,

Daegu 41931, Korea

Tel: +82-53-250-7831

Fax: +82-53-250-7840

E-mail: neurocho@gmail.com
Rapid eye movement (REM) sleep behavior disorder (RBD) is a parasomnia characterized by sleep interruption or trauma due to abnormal behaviors that occur during REM sleep. The pathophysiology of RBD is known to be a dysfunction of brainstem circuit that causes the loss of skeletal muscle atonia during REM sleep. The diagnosis of RBD is needed to confirm REM sleep without atonia in the polysomnography. The management of RBD includes not only drug treatment, but also to prevent injury from RBD and to followup on neurodegenerative diseases that may occur later. RBD is thought to be a prodromal stage of neurodegenerative disease associated with $\alpha$-synucleoinopathy, such as Parkinson's Disease or multiple system atrophy. This article reviews the symptoms, epidemiology, diagnosis and treatment of RBD, the relevance of neurodegenerative diseases, and recent research trends.

J Sleep Med 2018;15(1):1-7 Dream enactment behavior, Neurodegenerative disease.

\section{서 론}

렘수면 행동 장애[rapid eye movement(REM) sleep behavior disorder, RBD]는 렘수면(REM sleep) 중에 발생하는 비정 상적인 움직임을 특징으로 하는 사건수면(parasomnia) 중 하 나이다.

이 질환은 Schenck 등에 의해 1986년에 처음 보고되었으 며, 1987년부터 RBD라는 용어를 사용하였다. ${ }^{2,3}$ 이후 RBD에 대한 연구가 계속되어, 임상적 특징이 알려지게 되었으며, 진 단 기준이 성립되고, 치료 및 관리에 대한 방법도 보고되고 있다. 또한 RBD가 파킨슨병과 같은 신경퇴행성 질환과도 연 관이 있다는 사실이 알려지면서 관심이 더 많아지고 있다. ${ }^{4}$ 이 글에서는 현재까지 알려진 $\mathrm{RBD}$ 의 증상, 병리기전, 진단, 검사, 역학, 치료 방법과 최근 연구 동향 등에 대해 정리해 보 고자 한다.

This is an Open Access article distributed under the terms of the Creative Commons Attribution Non-Commercial License (https://creativecommons.org/licenses/by-nc/4.0) which permits unrestricted non-commercial use, distribution, and reproduction in any medium, provided the original work is properly cited.

\section{임상 양상}

$\mathrm{RBD}$ 의 가장 대표적인 증상은 수면 중 나타나는 이상 행동 인데, 이는 꿈의 내용을 옮기는 수면 관련 발성(sleep-related vocalization)과 복합적인 운동 행동(complex motor behavior) 으로 이루어져 있으며, 이로 인해 신체의 부상이나 수면 방해 (sleep interruption)를 일으키기도 한다.

운동 증상은 비특이적이고 반복적인 근육 경련부터 복잡 하고 목적을 지닌 것처럼 보이는 행동까지, 매우 다양한 양상 으로 나타날 수 있다. 손을 움켜쥐거나 팔을 휘두르고, 주먹 질을 하거나 발로 차는 행동을 보일 수 있고, 이 외에도 깨물 기, 침 뱉기, 머리카락 잡아당기기, 또는 공격으로부터 방어 하는 자세를 취하기도 한다. 이에 비해, 걷기나 방 밖으로 나 가는 행동은 드물게 나타난다. 성교와 비슷한 동작이나 먹고 마시는 행동, 배뇨나 배변 행위 등은 아주 드물게 보고되고 있다. ${ }^{5}$ 이런 행동들을 dream-enactment behavior(DEB)라고 부르며, 환자가 꿈에서 깨어날 때 그 꿈의 내용에 따라 목적 을 가지고 취한 행동의 형태로 나타난다. ${ }^{6}$

$\mathrm{RBD}$ 로 인해 다양한 상해를 입기도 하는데, 멍이나 피부 
가 찢어지는 정도부터, 치아가 깨지거나 얼굴이나 발가락뼈 골절, 척추나 엉치뼈, 허벅지뼈 골절과 같은 큰 골절이 발생 하기도 하며, 경막하 출혈이 발생하는 경우도 보고되어 있 다. 배우자를 보호하기 위해 침대를 따로 사용하거나, 침낭 사용, 자신의 몸을 로프로 묶거나, 아예 거실에서 수면을 취 하는 등의 방법을 사용하기도 한다는 환자들의 보고도 있었 다. 따라서 $\mathrm{RBD}$ 환자들의 삶의 질은 정상인에 비해 낮은데, 국내 $\mathrm{RBD}$ 환자들을 대상으로 한 연구에서는 건강한 일반인 들과 비교했을 때, 수면의 질이 낮고 우울감이 심하며, 전반 적인 삶의 질이 떨어지는 결과를 보였다. ${ }^{8}$

$\mathrm{RBD}$ 환자들에서 꿈의 내용의 공격성이 정상군에 비해 두 드러진다는 연구 보고가 있다. 꿈의 내용은 보통 매우 생생하 고, 폭력적이며, 낯선 사람과 싸우는 양상일 때가 많다고 한 다. 여성의 경우는 대결적이기 보다는 피해자로서 공격당하 는 내용일 때가 더 많아, 분노나 폭력성보다는 공포의 감정이 주로 나타난다고 하였다. ${ }^{9}$

\section{역 학}

선행 연구들에서는 일반 인구 중 RBD 유병률을 0.38 0.5\% 로 보고하고 있다. ${ }^{10,11}$ 한국 내에서의 유병률은 $2.01 \%$ 로 보고 된 바가 있어, 기존 연구들과 비교해 봤을 때 한국에서 $\mathrm{RBD}$ 유병률이 상대적으로 높다고 볼 수 있지만, 인종에 따른 유병 률은 추가 연구가 필요하겠다. ${ }^{12}$

$\mathrm{RBD}$ 의 역학적 특성 중 대표적인 점은 남성에서 좀 더 많 이 발병한다는 점과 주로 50세 이후에 발병한다는 점이다. 50 세 이전에 발병하는 $\mathrm{RBD}$ 의 경우는 덜 난폭한 양상을 보이고, 항우울제 사용 빈도가 좀 더 많이 보고되며, 기면증(narcolepsy)과 병발되는 경우도 있다고 보고되어 있다..$^{13-16}$ 또한 신 경퇴행성 질환으로의 진행이 50 세 이전 발병군에서는 적은 것으로 알려져 있다. ${ }^{17}$

\section{병리기전}

고양이와 쥐를 이용하여 시행한 동물 실험 결과에 따르면, 두 종류의 운동 시스템이 렘수면에 연관되어 있을 것으로 제 시되고 있다. ${ }^{18}$ 하나는 렘수면무긴장소실(REM sleep without atonia, RSWA)에 연관되는 시스템으로 알려져 있으며, 다른 하나는 locomotor generator로 알려져 있으나 locomotor generator에 대해서는 명확하게 밝혀져 있지 않다. ${ }^{19}$ 렘수면 무긴장소실과 연관되어 있을 것으로 생각되는 부위는 precoeruleus와 sublaterodorsal(SLD) nucleus이다. ${ }^{20}$ 정상적인 렘 수면 상태에서는 이 부위들이 직접 및 간접 경로를 통해 억제
회로를 활성화시키고, 이로 인해 운동신경 활성이 억제되어 렘수면 시의 근긴장 저하 및 소실 상태가 나타나게 된다. 구 조적 병변이나 퇴행성 변화, 약물 등으로 인해 이 SLD 부분 에 이상이 생기면 렘수면무긴장소실이 나타나는 것으로 추 론된다(Fig. 1).

\section{진 단}

2014년에 발표된 국제수면질환분류 기준 3판(The International Classification of Sleep Disorders, 3rd edition)의 RBD 진단 기준에 따르면, 2판에 이어 수면다원검사(polysomnography, PSG)에서 근전도상 렘수면무긴장소실이 관찰되 어야 한다고 기술되어 있으나, 2판에 비해서는 전반적으로 간소화된 진단 기준을 보여주고 있으며 그 내용은 Table 1과 같다. ${ }^{1} \mathrm{PSG}$ 에서 RSWA를 판단하기 위해서 미국수면학회에 서 수면판독기준(The American Academy of Sleep Medicine manual for the scoring of sleep and associated events) version 2.4에 나와 있는 기준을 사용하며, 그 내용은 Table 2에 정리되어 있다. ${ }^{21}$ 하지만, 현재까지 렘수면무긴장소실을 측정 하기 위한 최선의 방법에 대한 표준화는 이루어지지 않은 상 태이다. 미국수면학회에서 수면판독기준에 따른 렘수면무긴 장소실 측정 방법은 Table 2 및 Fig. 2와 같다. 국내에서는 기 존의 에폭 단위 도수적 분석 대신에 컴퓨터 지원 방식을 이용

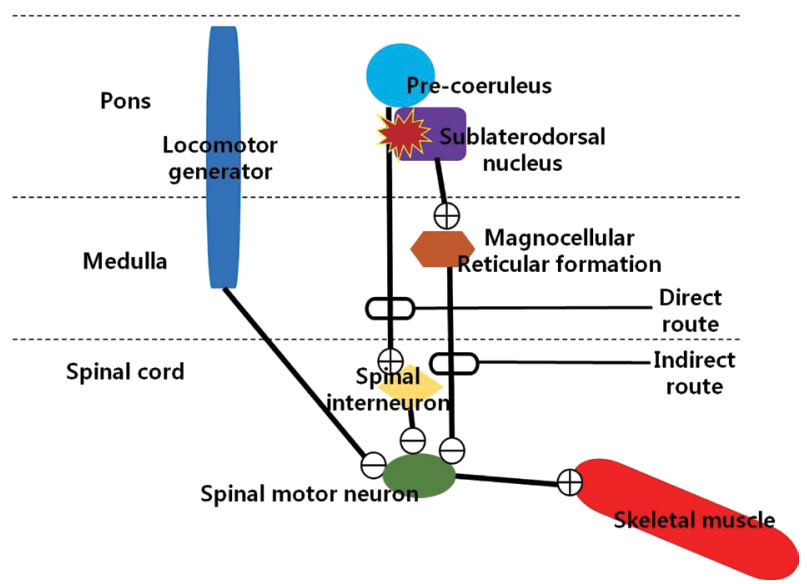

Figure 1. Pathophysiology of RBD. In normal physiology of REM sleep with atonia, precoeruleus and SLD nucleus activate two inhibitory pathways (direct and indirect route). The SLD nucleus stimulates an inhibitory spinal interneuron through the direct route which inhibits or hyperpolarizes the motor neuron to produce skeletal muscle atonia. The SLD nucleus activates the MCRF via the indirect pathway that causes MCRF to inhibit motor neurons to produce atonia of skeletal muscle. However, the skeletal muscle may be activated during sleep due to the SLD nucleus and/or afferent or efferent pathway dysfunction which normally inhibits motor neurons in RBD. ${ }^{18} \mathrm{RBD}$ : REM sleep behavior disorder, REM: rapid eye movement, SLD: sublaterodorsal, MCRF: medullary cell reticular formation. 
Table 1. Diagnostic criteria of RBD (International Classification of Sleep Disorders, 3rd edition)

Criteria A-D must be met

A. Repeated episodes of sleep related vocalization and/or complex motor behaviors

B. These behaviors are documented by PSG to occur during REM sleep, or based on clinical history of dream enactment, are presumed

to occur during REM sleep

C. Polysomnographic recording demonstrates RWA

D. The disturbance is not better explained by another sleep disorder, mental disorder, medication, or substance use

1) This criterion can be fulfilled by observation of repetitive episodes during a single night of video PSG, 2) The observed vocalizations or behaviors often correlate with simultaneously occurring dream mentation, leading to the frequent report of "acting out one's dreams", 3) As defined by the guidelines for scoring PSG features of RBD in the most recent version of the American Academy of Sleep Medicine manual for scoring of sleep associated events, 4) Upon awakening, the individual is typically awake, alert, coherent, and oriented, 5) On occasion, there may be patients with a typical clinical history of RBD with dream-enacting behaviors, who also exhibit typical RBD behaviors during vPSG, but do not demonstrate sufficient RWA, based on the current evidence-based data, to satisfy the PSG criteria for diagnosing RBD. In such patients, RBD may be previsionally diagnosed, based on clinical judgment. The same rule applies when vPSG is not readily available. 6) Medications may unmask latent RBD with preexisting RWA, according to current expert opinion. Therefore, medication-induced RBD can be diagnosed as RBD, using clinical judgment, pending future longitudinal studies. RBD: REM sleep behavior disorder, REM: rapid eye movement, PSG: polysomnography, RWA: REM sleep without atonia, vPSG: video-PSG

Table 2. Scoring PSG features of RBD (American Academy of Sleep Medicine ver. 2.4)

1. Score in accordance with following definitions: recommended

A. Sustained muscle activity (tonic activity) in REM sleep: an epoch of REM sleep with at least $50 \%$ of the duration of the epoch having chin EMG amplitude greater than the minimum amplitude demonstrated in NREM sleep

B. Excessive transient muscle activity (phasic activity): in which a 30-second epoch of REM sleep divided into 10 sequential 3-second mini-epochs, at least 5 (50\%) of mini-ephocs contain bursts of transient muscle activity. In RBD, excessive transient muscle activity bursts are $0.1-5 \mathrm{~s}$ in duration and at least 4 times as high in amplitude as the background EMG activity

2. The polysomnographic characteristics of RBD are characterized by either or both of the following features: recommended

A. Sustained muscle activity in REM sleep in the chin EMG

B. Excessive transient muscle activity during REM in the chin or limb EMG

1) Transient muscle activity and occasional accompanying visible twitching small muscle groups are a normal phenomenon seen in REM sleep. When larger muscle groups are involved, this activity is not associated with large, overt muscular activity acting across large joints. When smaller muscle groups are involved, the movement often involves the distal muscles the hands and face or the corners of the mouth. Transient muscle activity may be excessive in RBD, 2) The sustained muscle activity and the excessive transient muscle activity observed in REM sleep may be interrupted by superimposed (usually dream-enacting) behaviors in RBD, 3) In normal individuals there is an atonia seen in REM sleep in the chin and anterior tibialis EMG. In this state the baseline amplitude of the EMG signal decreased markedly. This atonia of REM sleep is lost to a considerable extent in RBD, with variable frequency, and as a result, the EMG baseline amplitude is often higher. In this situation, the EMG can be said to be in a tonic rather than atonic state. PSG: polysomnography, RBD: REM sleep behavior disorder, REM: rapid eye movement, EMG: electromyography, NREM: non-REM

한 렘수면무긴장소실 정량화 분석 방법에 대한 연구가 있었 으며,22 에폭 단위가 아닌 전체 PSG data에서 렘수면무긴장소 실을 정량적 분석하는 방법에 대해서도 고려될 필요가 있겠다. $\mathrm{RBD}$ 진단을 위해서는 $\mathrm{PSG}$ 검사가 필요하지만, $\mathrm{PSG}$ 검사 는 비용이 많이 들고 검사실에서 하룻밤을 자야 하는 번거로 움이 있다. 따라서, 수면장애 환자 문진 시 간편하게 평가하는 스크리닝 검사 도구가 필요하다. 최근에는 REM Sleep Behavior Disorder Screening Questionnaire-Hong Kong(RB$\mathrm{DQ}-\mathrm{HK})$ 이 한국어로 번역되어 유효성 확인 연구가 완료되었 으며, $\mathrm{RBD}$ 스크리닝에 있어 높은 민감도와 특이도를 보여 임 상에서 유용하겠다. ${ }^{23}$

신경학적으로 정상인 환자에게 $\mathrm{RBD}$ 진단을 위한 뇌 자기 공명영상(magnetic resonance image) 검사 시행이 반드시 필 요하지는 않다. 그러나 $\mathrm{RBD}$ 를 일으킬 만한 이상 소견이 영
상 검사에서 발견된 경우에는 lesional $\mathrm{RBD}$ 라고 진단할 수 있다.

수면 중 나타나는 이상 행동으로 대표적인 감별 진단인 경 련(seizure)과 감별을 위해 뇌파 검사(electroencephaogram) 의 시행도 중요하다. 특히, 젊은 나이에 발생하고 증상이 정 형화되어 있고, 양상을 기억하지 못하는 경우에는 뇌파 검사 가 반드시 시행되어야 하며, 대사성 혹은 독성 뇌병증, 뇌염, 또는 lesional $\mathrm{RBD}$ 의 경우에도 뇌파 검사가 시행되면 감별에 도움이 된다.

그 외, 감별 진단으로는 $\mathrm{DEB}$ 가 관찰될 수 있는 질환들을 고려해야 하며, 야경증(sleep terror)과 같은 비렘수면장애 (non-REM sleep parasomnia), 수면 무호흡증(obstructive sleep apnea, OSA)이나 기면증(narcolepsy)에서 관찰되는 렘 수면무긴장소실, 약물이나 술을 중단해서 발생하거나 혹은 

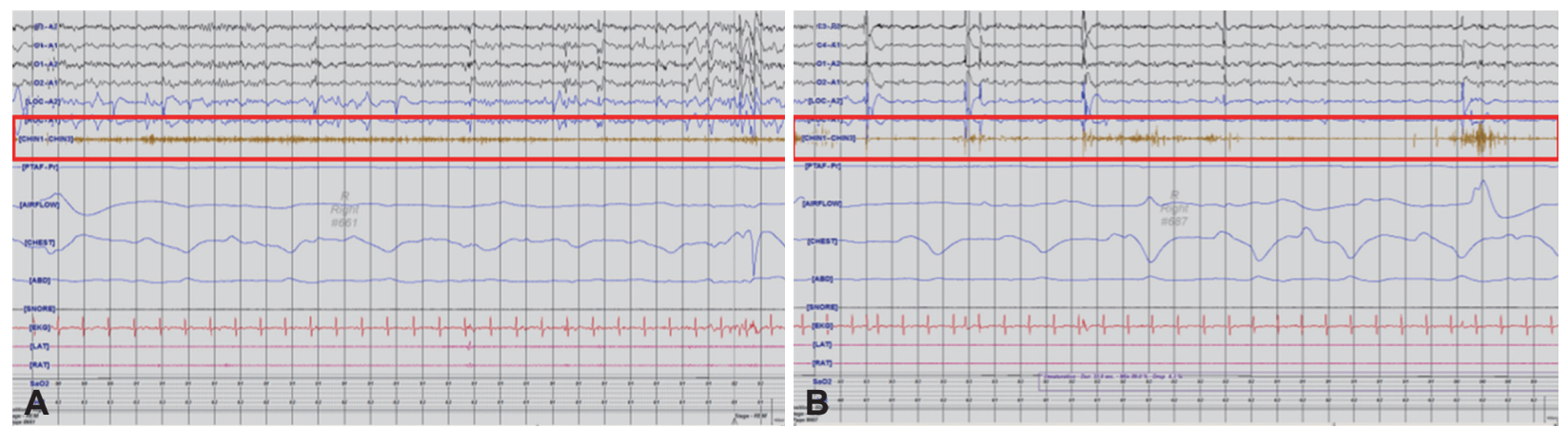

Figure 2. Scoring the REM sleep without atonia in polysomnography. (A) An epoch of REM sleep with at least $50 \%$ of the duration of the epoch having chin EMG amplitude scored as sustained muscle activity (tonic activity). (B) In which a 30-second epoch of REM sleep divided into 10 sequential 3-second mini-epochs, at least 5 (50\%) of mini-ephocs contain bursts of transient muscle activity scored as excessive transient muscle activity (phasic activity) in chin EMG. REM: rapid eye movement, EMG: electromyogrpahy.

뇌의 구조적 이상에 의해 발생하는 lesional $\mathrm{RBD}$ 와 같은 이 차성 RBD 등을 모두 고려해야 한다. 이 외에도 섬망이나 치 매, 악몽, 출면기 환각, 주기성 사지 운동 등도 RBD 진단 시에 감별해야 할 질환들이다. ${ }^{24} \mathrm{OSA}$ 로 인해 발생하는 $\mathrm{DEB}$ 의 경 우에는, continuous positive airway pressure 치료를 통해 $\mathrm{RBD}$ 증상이 호전된 경우도 알려져 있다. ${ }^{9}$

약물 복용에 의해서도 RBD 증상이 나타날 수 있다고 알려 져 있다. 대표적인 약제로는 항우울제가 있으며, 이 중에서도 선택적 세로토닌 재흡수 억제제(fluoxetine, paroxetin), 세로 토닌 노르아드레날린 재흡수 억제제(venlafaxine, mirtazapine), 삼환계 항우울제 등이 $\mathrm{RBD}$ 증상을 유발한다고 잘 알려 져 있다. ${ }^{25-28}$ 항우울제는 기저의 우울증이나 퇴행성 뇌질환과 상관 없이 렘수면 중의 근긴장도를 증가시킨다는 연구 결과 도 있었다. ${ }^{29}$ 또한 selegiline, rivastigmine, atenolol, opioid, 그 리고 카페인 사용 등과도 관련되어 있다고 보고되어 있다. ${ }^{30-32}$

\section{신경퇴행성 질환과의 연관성}

$\mathrm{RBD}$ 가 증상을 일으킬 만한 이차성 병변이나 특정 병인이 없는 상태에서 발생하는 경우에 특발성 RBD(idiopathic RBD, $\mathrm{iRBD}$ )라고 한다. 특발성 $\mathrm{RBD}$ 가 신경퇴행성 질환과 연관되어 선행한다고 알려져 있는데, 특히 파킨슨병(Parkinson's disease, $\mathrm{PD}$ ), 다계통 위축증(multiple system atrophy, MSA), 또는 루 이소체치매(dementia with Lewy body)와 같은 $\alpha$-synucleinopathy와의 연관성이 많은 연구에서 보고되어 있다. ${ }^{33-36}$ MSA 환자의 90 100\%, dementia with Lewy bodies(DLB) 환자의 50 80\%, 그리고 $\mathrm{PD}$ 환자의 경우에는 16 47\%에서 $\mathrm{RBD}$ 가 동반된다고 알려져 있다. ${ }^{1,37}$ 2013년에 발표된 연구에 따르면, 16 년 동안 추적 관찰한 결과, 특발성 $\mathrm{RBD}(\mathrm{iRBD})$ 환자 의 $81 \%$ 에서 파킨슨증이나 치매 증상이 발생했으며, $\mathrm{iRBD}$ 에 서 파킨슨 질환이 나타나기까지의 평균 기간은 14 년이었다. ${ }^{38}$
최근 들어 질병-조절 치료(disease-modifying therapy)에 대한 관심이 늘어나면서 $\mathrm{PD}$ 전구기(prodromal $\mathrm{PD}$ )의 중요 도 역시 함께 증가하고 있으며, 이에 prodromal $\mathrm{PD}$ 의 표지자 중 하나로 주목받고 있는 RBD 환자군을 대상으로 한 코호트 연구들이 지역별로 진행되고 있다. ${ }^{39}$ 더 나아가 prodromal $\mathrm{RBD}$ (렘수면무긴장소실 혹은 렘수면 관련 사건이 있으나 $\mathrm{RBD}$ 진단 기준을 만족시키지 못하는 상태)라는 개념도 등장했으며, 이런 증상이 있는 사람들이 $\mathrm{RBD}$ 를 거쳐 $\alpha$-synucleinopathy 로 진행할 가능성이 높기에 신경퇴행성 변화를 조기에 스크 리닝하는 단계로 고려해야 한다는 의견도 제시되고 있다. 47

$\mathrm{RBD}$ 의 진단이 신경퇴행성 질환의 예후를 예측하는 인자 로서도 작용할 수 있는데, $\mathrm{PD}$ 환자에서 $\mathrm{RBD}$ 가 병발된 경우 에 질환의 중증도가 증가하며, 운동 동요(motor fluctuation) 현 상이 더 많이 나타나며, 변비와 기타 자율신경계 기능 이상이 더 심하게 발생한다고 알려져 있다. ${ }^{40}$ 한 연구에서는 RBD가 있는 $\mathrm{PD}$ 에서 경도인지장애(mild cognitive impairment)가 발 생할 가능성이 높으며, ${ }^{41}$ 또 다른 연구에서는 4.4 년 내에 치매 가 발생할 위험도가 증가한다는 결과도 보고된 바가 있다. ${ }^{42}$

이에 반해 tauopathy에서는 RBD가 드물게 동반되지만, 알 츠하이머 치매(Alzheimer's disease, $\mathrm{AD}$ ), 전두측두엽 치매 (fronto-temporal dementia), 진행성 핵상 마비(progresssive supranuclear palsy) 등에서도 나타난다고 하며, 이 외에도 척 수소뇌실조증(spinocerebellar ataxia), 헌팅톤병(Huntington's disease) 등에서도 RBD가 보고되어 있다. ${ }^{6,43-45}$

\section{신경영상검사의 활용}

$\mathrm{RBD}$ 의 질병 심각도 및 신경퇴행성 질환으로의 변화를 추 적 관찰하기 위한 방법으로 다양한 신경영상검사들이 활용 되고 있다. ${ }^{46}$ 시냅스 전 도파민 신경의 기능을 평가할 수 있는 도파민전달체영상(dopamine active transporter imaging)은 
흑색질줄무늬체(nigrostriatal) 도파민 신경 손상 정도를 파악 하고 그 퇴행을 예측하는 데 도움이 되며, ${ }^{47}$ 뇌관류 singlephoton emission computed tomography를 통한 뇌혈류 상태 분석을 통해 $\mathrm{RBD}$ 환자에서의 인지기능 저하와의 관련성을 보여줄 수도 있으며, $\mathrm{PD}$ 혹은 $\mathrm{DLB}$ 로의 진행을 예측하는 지 표로도 활용해 볼 수 있다. ${ }^{48}$ 뇌의 대사망(metabolic network) 분석을 통해서, $\mathrm{RBD}$ 가 $\mathrm{PD}, \mathrm{DLB}$, 혹은 $\mathrm{MSA}$ 로 진행하는 것 을 예측할 수 있다는 연구 결과들도 있다. ${ }^{49,50}$ 경두개 도플러 초음파(transcranial Doppler ultrasonography)는 흑색질의 퇴행성 변화를 추적 관찰하는 데 도움이 된다고 알려져 있 다. ${ }^{51}$ 이에 비해 뇌 자기공명영상을 이용한 연구들에서는 아 직까지 특징적인 결과가 나오지 않은 상태이다.

\section{치 료}

$\mathrm{RBD}$ 치료의 가장 중요한 목표는 환자 및 배우자(그 외 수 면 시 동반자)의 부상 발생을 사전에 방지하는 것이다. 이를 위해 수면 환경을 개선하거나 약물 치료를 시행하기도 하며, 증상을 유발하거나 악화시킬 수 있는 약제 사용을 피하는 것 이 필요하다. 치료 방법에 대해서는 Table 3에 정리되어 있다. $\mathrm{RBD}$ 증상으로 인한 부상 발생이 줄어들도록 다양한 방향으

Table 3. Clinical management of $\mathrm{RBD}^{52}$

\begin{tabular}{l} 
Pharmacotherapy \\
\hline Clonazepam: $0.5-2.0 \mathrm{mg} 30$ minutes before bedtime \\
Melatonin: $3-12 \mathrm{mg}$ hs \\
Others (uncertain efficacy) \\
Pramipexole, levodopa, paroxetine, clozapine, carbamazepine, \\
$\quad$ donepezil, rivastigmine, alprazolam, triazolam, \\
$\quad$ sodium oxybate, etc. \\
Rotigotine (only in Parkinson's disease patient) \\
\hline Safe sleep environment \\
\hline Avoid sleeping beside window \\
Pad the edge of furniture \\
Remove potentially dangerous objects \\
Sleep on the mattress \\
Separate bed partner until RBD has been controlled \\
\hline Avoid or discontinue of medications reported to trigger or \\
aggravate RBD \\
\hline Selective serotonin reuptake inhibitors: fluoxetine, paroxetine \\
Serotonin-norepinephrine reuptake inhibitors: venlafaxine, \\
mirtazapine \\
Tricyclic antidepressants \\
Monoamine oxidase inhibitors: selegiline \\
Beta-blocker: atenolol \\
Acetylcholine esterase inhibitor: rivastigmine \\
\hline RBD: REM sep behavior disorder REM: rapid eye movement
\end{tabular}

로 수면 환경을 개선하는 것이 도움이 된다. 종양, 뇌염 등과 같이 $\mathrm{RBD}$ 를 일으킬 만한 기저 원인이 확실히 있는 경우에는 그에 대한 치료가 선행되는 것이 필요하다. $\mathrm{RBD}$ 의 약물 치료 에 대해서는 아직 대규모의 무작위 대조 시험(randomized controlled trial) 결과가 없는 상태이나, 증상 치료에 사용해 볼 수 있다고 알려진 가장 대표적인 약제는 clonazepam과 melatonin이다. ${ }^{52}$ Clonazepam은 $0.5 \mathrm{mg}$ 에서 $2 \mathrm{mg}$ 까지 잠들기 30 분 전에 복용하는 것이 권고된다. Clonazepam은 장시간 작용 benzodiazepine계 약물 중 하나로, $\mathrm{RBD}$ 에 의한 행동 장애를 조절하고 나쁜 꿈의 비율도 줄여주는 효과가 높으며, 상대적으로 부작용이 적고 의존성이 낮아 장기간 사용하기 용이하다. 그러나 OSA 증상을 일으킬 수 있으므로 OSA 환 자에게 처방 시에는 유의해야 하며, 이 외에도 치매 환자 및 보행 장애가 있는 환자에게서 인지기능 저하와 자세 유지 균 형 장애를 악화시킬 수 있다고 알려져 있으므로 처방에 주의 를 기울여야 한다. Melatonin은 RBD의 치료 효과도 좋으며 부작용이 적고 안전하다는 장점이 있다. 작용기전은 불명확 하나 렘수면무긴장소실을 줄인다고 알려져 있다. 보통 $3 \mathrm{mg}$ 에서 $12 \mathrm{mg}$ 의 용량으로 잠자기 전에 사용해 볼 수 있다. 부작 용으로는 아침 시간대의 두통, 어지럼증, 진정 등이 있다..$^{53}$

Donepezil과 rivastigmine과 같은 acetylcholine esterase 억제제가 $\mathrm{AD}$ 혹은 $\mathrm{DLB}$ 환자에서 $\mathrm{RBD}$ 증상 조절에 도움이 된다는 증례 보고 및 연구 결과가 있었다. 54,55 그러나 rivastigmine이 $\mathrm{AD}$ 환자에서 $\mathrm{RBD}$ 증상을 유발시켰다는 증례 보고 도 있었다. ${ }^{31}$

Pramipexole은 소규모의 RBD 환자를 대상으로 한 연구 에서 증상 조절에 효과가 있다는 보고가 있었으나, ${ }^{56,57} \mathrm{PD}$ 와 $\mathrm{RBD}$ 증상이 같이 있는 환자를 대상으로 한 다른 연구에서는 파킨슨병의 증상은 호전되나 $\mathrm{RBD}$ 증상은 호전이 없다는 보 고가 있었다. ${ }^{58}$ Rotigotine은 PD 환자를 대상으로 한 소규모 연구가 있으며, $\mathrm{RBD}$ 증상을 부분적으로 호전시킨다는 결과 보고가 있었다. ${ }^{59}$

Quetiapine과 같은 비전형 항정신병약물들의 경우에도 $\mathrm{RBD}$ 증상을 호전시켰다는 연구 결과도 있으나, $\mathrm{DLB}$ 환자에 게 사용할 경우에는 신경이완제 과민증(neuroleptic hypersensitivity) 등의 부작용이 발생할 수 있으므로 주의가 필요 하다. ${ }^{6}$

위와 같은 약제들 외에 carbamazepine, levodopa, paroxetine, alprazolam 등의 약제들이 RBD 증상을 호전시킨다는 연구 결과들이 있었으나, 데이터가 불충분하여 치료 약제로 권고하기엔 근거가 부족한 상태이다. ${ }^{2}$

RBD: REM sleep behavior disorder, REM: rapid eye movement 


\author{
결 론 \\ $\mathrm{RBD}$ 는 1980년대 이후로 그 임상적 특성과 진단, 그리고 \\ 치료에 대해 많은 연구가 진행되고 있다. 특히 $\mathrm{PD}$ 와 같은 신 \\ 경퇴행성 질환과의 연관성이 알려지면서 그 중요성이 더욱 \\ 강조되고 있다. 그러나 아직도 정확한 발병기전과 병태 생리 \\ 에 대한 정보가 부족하며, 치료 방법 역시 제한적이다. 또한 \\ 신경퇴행성 질환으로 진행되는 과정에 대한 연구 역시 계속 \\ 필요한 상태이다. 현재 한국은 고령화가 가속화되고 있으므 \\ 로 RBD에 대한 관심과 이해가 매우 중요하다.
}

\section{REFERENCES}

1. American Academy of Sleep Medicine. International classification of sleep disorders, 3rd ed. Darien: American Academy of Sleep Medicine, 2014.

2. Schenck CH, Bundlie SR, Ettinger MG, Mahowald MW. Chronic behavioral disorders of human REM sleep: a new category of parasomnia. Sleep 1986;9:293-308.

3. Schenck CH, Bundlie SR, Patterson AL, Mahowald MW. Rapid eye movement sleep behavior disorder. A treatable parasomnia affecting older adults. JAMA 1987;257:1786-1789.

4. Iranzo A, Santamaria J, Tolosa E. Idiopathic rapid eye movement sleep behaviour disorder: diagnosis, management, and the need for neuroprotective interventions. Lancet Neurol 2016;15:405-419.

5. Oudiette D, De Cock VC, Lavault S, Leu S, Vidailhet M, Arnulf I. Nonviolent elaborate behaviors may also occur in REM sleep behavior disorder. Neurology 2009;72:551-557.

6. Boeve BF. REM sleep behavior disorder: updated review of the core features, the REM sleep behavior disorder-neurodegenerative disease association, evolving concepts, controversies, and future directions. Ann N Y Acad Sci 2010;1184:15-54.

7. McCarter SJ, St Louis EK, Boswell CL, et al. Factors associated with injury in REM sleep behavior disorder. Sleep Med 2014;15:1332-1338.

8. Kim KT, Motamedi GK, Cho YW. Quality of life in patients with an idiopathic rapid eye movement sleep behaviour disorder in Korea. $J$ Sleep Res 2017;26:422-427.

9. Fernández-Arcos A, Iranzo A, Serradell M, Gaig C, Santamaria J. The clinical phenotype of idiopathic rapid eye movement sleep behavior disorder at presentation: a study in 203 consecutive patients. Sleep 2016;39:121-132.

10. Ohayon MM, Caulet M, Priest RG. Violent behavior during sleep. J Clin Psychiatry 1997;58:369-376; quiz 377.

11. Chiu HF, Wing YK, Lam LC, et al. Sleep-related injury in the elderly-an epidemiological study in Hong Kong. Sleep 2000;23:513-517.

12. Kang SH, Yoon IY, Lee SD, Han JW, Kim TH, Kim KW. REM sleep behavior disorder in the Korean elderly population: prevalence and clinical characteristics. Sleep 2013;36:1147-1152.

13. Bonakis A, Howard RS, Williams A. Narcolepsy presenting as REM sleep behaviour disorder. Clin Neurol Neurosurg 2008;110:518-520.

14. Nightingale S, Orgill JC, Ebrahim IO, de Lacy SF, Agrawal S, Williams AJ. The association between narcolepsy and REM behavior disorder (RBD). Sleep Med 2005;6:253-258.

15. Ju YE, Larson-Prior L, Duntley S. Changing demographics in REM sleep behavior disorder: possible effect of autoimmunity and antidepressants. Sleep Med 2011;12:278-283.

16. Ju YE. Rapid eye movement sleep behavior disorder in adults younger than 50 years of age. Sleep Med 2013;14:768-774.

17. Bonakis A, Howard RS, Ebrahim IO, Merritt S, Williams A. REM sleep behaviour disorder (RBD) and its associations in young patients.
Sleep Med 2009;10:641-645.

18. Boeve BF, Silber MH, Saper CB, et al. Pathophysiology of REM sleep behaviour disorder and relevance to neurodegenerative disease. Brain 2007;130(Pt 11):2770-2788.

19. Morrison AR. The pathophysiology of REM-sleep behavior disorder. Sleep 1998;21:446-449.

20. Rodriguez CL, Jaimchariyatam N, Budur K. Rapid eye movement sleep behavior disorder: a review of the literature and update on current concepts. Chest 2017;152:650-662.

21. Berry RB, Brooks R, Gamaldo CE, et al. The AASM manual for the scoring of sleep and associated events: rules, terminology and technical specifications: version 2.3. Darien: American Academy of Sleep Medicine, 2015.

22. Lee JH, Jung YJ, Cha HK, Rhee HY, Choi HY, Shin WC. Quantification of REM sleep without atonia in Korean REM sleep behavior disorder patients: comparison of manual and computer-assisted scoring methods. J Korean Sleep Res Soc 2014;11:50-56.

23. You S, Moon HJ, Do SY, et al. The REM sleep behavior disorder screening questionnaire: validation study of the Korean version (RBDQ-KR). J Clin Sleep Med 2017;13:1429-1433.

24. Schenck CH, Milner DM, Hurwitz TD, Bundlie SR, Mahowald MW. A polysomnographic and clinical report on sleep-related injury in 100 adult patients. Am J Psychiatry 1989;146:1166-1173.

25. Schenck CH, Mahowald MW, Kim SW, O'Connor KA, Hurwitz TD. Prominent eye movements during NREM sleep and REM sleep behavior disorder associated with fluoxetine treatment of depression and obsessive-compulsive disorder. Sleep 1992;15:226-235.

26. Parish JM. Violent dreaming and antidepressant drugs: or how paroxetine made me dream that I was fighting Saddam Hussein. J Clin Sleep Med 2007;3:529-531.

27. Postuma RB, Gagnon JF, Tuineaig M, et al. Antidepressants and REM sleep behavior disorder: isolated side effect or neurodegenerative signal? Sleep 2013;36:1579-1585.

28. Schenck CH, Mahowald MW. Motor dyscontrol in narcolepsy: rapideye-movement (REM) sleep without atonia and REM sleep behavior disorder. Ann Neurol 1992;32:3-10.

29. McCarter SJ, St Louis EK, Sandness DJ, et al. Antidepressants increase REM sleep muscle tone in patients with and without REM sleep behavior disorder. Sleep 2015;38:907-917.

30. Louden MB, Morehead MA, Schmidt HS. Activation by selegiline (Eldepryle) of REM sleep behavior disorder in parkinsonism. WV Med J 1995;91:101.

31. Yeh SB, Yeh PY, Schenck CH. Rivastigmine-induced REM sleep behavior disorder (RBD) in a 88-year-old man with Alzheimer's disease. J Clin Sleep Med 2010;6:192-195.

32. Wong JC, Li J, Pavlova M, et al. Risk factors for probable REM sleep behavior disorder: a community-based study. Neurology 2016;86:13061312.

33. Adler CH, Hentz JG, Shill HA, et al. Probable RBD is increased in Parkinson's disease but not in essential tremor or restless legs syndrome. Parkinsonism Relat Disord 2011;17:456-458.

34. Palma JA, Fernandez-Cordon C, Coon EA, et al. Prevalence of REM sleep behavior disorder in multiple system atrophy: a multicenter study and meta-analysis. Clin Auton Res 2015;25:69-75.

35. Boeve BF, Silber MH, Ferman TJ, et al. REM sleep behavior disorder and degenerative dementia: an association likely reflecting Lewy body disease. Neurology 1998;51:363-370.

36. Iranzo A, Fernández-Arcos A, Tolosa E, et al. Neurodegenerative disorder risk in idiopathic REM sleep behavior disorder: study in 174 patients. PLoS One 2014;9:e89741.

37. Högl B, Stefani A, Videnovic A. Idiopathic REM sleep behaviour disorder and neurodegeneration-an update. Nat Rev Neurol 2018;14:4055.

38. Schenck CH, Boeve BF, Mahowald MW. Delayed emergence of a parkinsonian disorder or dementia in $81 \%$ of older men initially diagnosed 
with idiopathic rapid eye movement sleep behavior disorder: a 16-year update on a previously reported series. Sleep Med 2013;14:744-748.

39. Barber TR, Lawton M, Ben-Shlomo Y, Hu MTM. Considerations in the use of MDS research criteria for prodromal Parkinson's in rapid eye movement sleep behaviour disorder and population cohorts. Sleep 2017;40.

40. Nihei Y, Takahashi K, Koto A, et al. REM sleep behavior disorder in Japanese patients with Parkinson's disease: a multicenter study using the REM sleep behavior disorder screening questionnaire. J Neurol 2012;259:1606-1612.

41. Gagnon JF, Vendette M, Postuma RB, et al. Mild cognitive impairment in rapid eye movement sleep behavior disorder and Parkinson's disease. Ann Neurol 2009;66:39-47.

42. Anang JB, Gagnon JF, Bertrand JA, et al. Predictors of dementia in Parkinson disease: a prospective cohort study. Neurology 2014;83:12531260 .

43. Wang P, Wing YK, Xing J, et al. Rapid eye movement sleep behavior disorder in patients with probable Alzheimer's disease. Aging Clin Exp Res 2016;28:951-957.

44. McCarter SJ, St Louis EK, Boeve BF. Sleep disturbances in frontotemporal dementia. Curr Neurol Neurosci Rep 2016;16:85.

45. Velázquez-Pérez LC, Rodríguez-Labrada R, Fernandez-Ruiz J. Spinocerebellar ataxia type 2: clinicogenetic aspects, mechanistic insights, and management approaches. Front Neurol 2017;8:472.

46. Heller J, Brcina N, Dogan I, et al. Brain imaging findings in idiopathic REM sleep behavior disorder (RBD)-A systematic review on potential biomarkers for neurodegeneration. Sleep Med Rev 2017;34:23-33.

47. Iranzo A, Valldeoriola F, Lomeña F, et al. Serial dopamine transporter imaging of nigrostriatal function in patients with idiopathic rapid-eyemovement sleep behaviour disorder: a prospective study. Lancet Neurol 2011;10:797-805.

48. Dang-Vu TT, Gagnon JF, Vendette M, Soucy JP, Postuma RB, Montplaisir J. Hippocampal perfusion predicts impending neurodegeneration in REM sleep behavior disorder. Neurology 2012;79:2302-2306.
49. Wu P, Yu H, Peng S, et al. Consistent abnormalities in metabolic network activity in idiopathic rapid eye movement sleep behaviour disorder. Brain 2014;137(Pt 12):3122-3128.

50. Holtbernd F, Gagnon JF, Postuma RB, et al. Abnormal metabolic network activity in REM sleep behavior disorder. Neurology 2014;82:620627.

51. Iranzo A, Stockner H, Serradell M, et al. Five-year follow-up of substantia nigra echogenicity in idiopathic REM sleep behavior disorder. Mov Disord 2014;29:1774-1780.

52. Aurora RN, Zak RS, Maganti RK, et al.; Standards of Practice Committee; American Academy of Sleep Medicine. Best practice guide for the treatment of REM sleep behavior disorder (RBD). J Clin Sleep Med 2010;6:85-95.

53. Kunz D, Mahlberg R. A two-part, double-blind, placebo-controlled trial of exogenous melatonin in REM sleep behaviour disorder. J Sleep Res 2010;19:591-596.

54. Ringman JM, Simmons JH. Treatment of REM sleep behavior disorder with donepezil: a report of three cases. Neurology 2000;55:870-871.

55. Grace JB, Walker MP, McKeith IG. A comparison of sleep profiles in patients with dementia with lewy bodies and Alzheimer's disease. Int $J$ Geriatr Psychiatry 2000;15:1028-1033.

56. Sasai T, Inoue Y, Matsuura M. Effectiveness of pramipexole, a dopamine agonist, on rapid eye movement sleep behavior disorder. Tohoku J Exp Med 2012;226:177-181.

57. Schmidt MH, Koshal VB, Schmidt HS. Use of pramipexole in REM sleep behavior disorder: results from a case series. Sleep Med 2006;7: 418-423.

58. Kumru H, Iranzo A, Carrasco E, et al. Lack of effects of pramipexole on REM sleep behavior disorder in Parkinson disease. Sleep 2008;31:14181421.

59. Wang Y, Yang Y, Wu H, Lan D, Chen Y, Zhao Z. Effects of rotigotine on REM sleep behavior disorder in Parkinson disease. J Clin Sleep Med 2016;12:1403-1409. 